\title{
Research on Interior Space of Ecological Building Complex Based on the Demand of Entrepreneurial Group*
}

\author{
Yuqi Zhuang \\ College of Art and Design \\ Beijing Forestry University \\ Beijing, China
}

\author{
Zhongying Shi \\ College of Art and Design \\ Beijing Forestry University \\ Beijing, China
}

\begin{abstract}
In recent years, the building complex of China's economically developed cities has formed a certain scale of development. Due to the larger scale of development of the current building complex and the complexity of the internal functional space composition and space requirements, it is extremely important to correctly solve the complex spatial relationship between various functions. The ecological building complex is a building complex constructed as per the needs of entrepreneurial groups and organically combined with other diverse functional spaces depended on each other. This has become an important mode for the extensive development of building complexes in modern cities. This highly concentrated architectural form conforming to the needs of entrepreneurial groups can not only significantly improve the various disadvantages brought about by the unilateral emphasis on functional zoning of modern urban development modes, but also facilitate the integration of new demands for uninterrupted urban performance development. The building space of a building complex based on the needs of entrepreneurial groups can be simply summarized into two main components: commercial building space and residential building space. When the location of the building complex in the urban function and its main functional space composition are defined, it is needed to further scientifically design its internal space combination. This paper discusses the interior space design of building complex according to the needs of entrepreneurial groups.
\end{abstract} group

Keywords-interior space; building complex; entrepreneurial

\section{INTRODUCTION}

\section{A. Research Background}

In the covering range of the city's commercial center which has the highest population density and the largest flow of people, architecture has become a place for people to communicate and perform daily work activities, rather than just a single function space in the traditional sense. As the main component of the city's central business district, urban buildings have become increasingly prominent in the city, and have become synonymous with urban business culture

*Fund: A Special Teaching Study on The Direct Introduction of Animal and Plant Factors in Art Design (Project No. BJFU2018JY112). and a direct reflection of the city's economic growth level in practice. Building complexes place higher demands on functional formats and space environments in order to break the pattern of operations and traditional trade structures. With its versatility, the building complex has become a symbol of modern cities and has gradually become one of the architectural categories closely related to the daily lives of urban residents. The "city" characteristics play an increasingly important role in the lives of urban people, changing urban spaces and even changing people's lifestyles.

In fact, the system mode of building complex design is more and more sound, and the design techniques and the expression forms are more and more diversified, but there are still outstanding problems. In terms of humanized design, there are still many shortcomings in the interior space of the complex. People tend to pay too much attention to commercial interests and reduce the concern for human nature. Because of the one-sided pursuit of functional space division and technical means, physical and mental needs are neglected. The topic of how to meet people's growing needs for life and spirit in the current era has raised widespread attention.

\section{B. Research Content}

The interior space design of the building complex based on the needs of entrepreneurial groups is the main content of the topic. The spatial composition, interface elements and sequence guidance of the interior space are studied in depth from the generation, classification, main features and development of the building complex. Through analyzing the main factors affecting the design of interior space, exploring the theoretical basis of interior space design and the inherent meaning of humanized design, finding out the problems in the design, raising thinking about the interior space environment of the building complex, and expecting the future development trend, practical and theoretical fundamentals for future design are provided.

\section{Research Significance}

As a kind of organization system in a certain area, the building complex supported by a new type of building mode and with multiple commercialization and versatility as its 
advantages, plays an important role in the economic development of the region and the entire city. Under the extensive and in-depth study of building complexes by relevant researchers at home and abroad, its structural system is constantly optimized and improved. Due to complex and varied social environment, so people's behavioral activities are also faced with more choices.

The guiding principles and implementation strategies of the humanized design of the interior space of the building complex have far-reaching implications for future design practice and theoretical research. It can highlight the peopleoriented design concept and improve the people's demand for life and spirit in today's social environment at maximum, thus improving the experience and satisfaction. Linking the unique historical background and cultural characteristics of the area with the humanized design of the interior space of the building complex creates a unique space environment, which let people to re-examine the excellent traditional culture to varying degrees. Strengthening humanized design on the basis of diversified needs, people's cultural identity and sense of belonging in the space environment can be deepened.

\section{Research Methods}

On the basis of collecting a large number of domestic and foreign related materials and literatures, combined with inductive method, example method, systematic analysis method and other various methods to carry out relevant analysis and research on this topic. In response to the characteristics of this topic, the following research methods have been adopted:

1) Literature analysis: According to the information of various professional books, journals and online databases, relevant information about this topic in the past 10 years at home and abroad and related data and picture information are collected and organized for analysis, comparison and induction. In the process of collection, the access to data channels is broadened, so targeted literature searches are conducted easily to extract content closely related to the subject, especially theoretical research related to architectural complexes and interior space design. Organizing and dissecting under the premise of understanding the main ideas, which constitutes the theoretical basis of the research of the topic.

2) Disciplinary synthesis: The interior design of the building complex is complex and diverse, involving not only the disciplines related to interior design, but also the profound influences on design disciplines such as design psychology, ergonomics, and environmental psychology. Only by systematically collating and performing multilevel analysis and research on the predecessors' theory and practical experience of different disciplines can we obtain more comprehensive analysis results.

3) Field research method: The most directly useful method in the on-site field research process is to obtain useful information through observation. By observing, photographing, and recording the target object, the firsthand information needed by the research institute can be obtained and the authenticity of the information is ensured. The problems in interior space design can be found out by conducting field surveys of representative buildings in Beijing, Shanghai, Tianjin and other cities in China through hands-on experience. So, systematically conduct comprehensive and in-depth research and summary can be conducted systematically.

4) Inductive deduction: The collected data is collated, researched, and analyzed in a number of different ways. The issues and points of attention that need to be addressed during the design process should be identified, and the root principles and strategies for interior design of building complexes can be explored.

\section{BASIC COMPOSITION OF INTERIOR SPACE}

\section{A. Type of Interior Spaces}

1) Open space: The open space is externally oriented which focus on communication with the space environment and emphasizes the integration of landscape with nature or surrounding space, so its privacy and restrictions are lower. It can present a wider indoor and outdoor landscape and can broaden the line of sight. Open spaces are more flexible when used, making it easier to change the interior layout. The open space is usually expressed as happy and positive from psychological effects. For example, glass curtain wall buildings, beachfront apartments, community centers, etc., are all open spaces.

2) Closed space: Surrounded by a certain height of enveloping entities, the closed space has a certain gap in vision and hearing, and will produce a sense of reliability, category and privacy from psychological effects. Spaces, such as bedrooms in private spaces, bathrooms, compartments in public spaces, and or independent studios, which all are separated by brick walls, concrete walls, etc., are enclosed spaces.

3) Semi-open space: When people work together, talk or hold other activities in a relatively empty space, they are often disturbed and lack certain privacy, and they can't feel intimately closed to each other. Although the closed space can alleviate the above deficiencies, it is also easy to cause feelings of depression, blockage and crowding. The semiopen space can solve the above shortcomings perfectly. It is a quadratic definition of space. It uses physical or symbolic means to define a small space in the original space, combining the closeness and the openness. This design method is widely used in many spaces. In the semi-open space, people can communicate with each other with a certain degree of privacy and domain, and bring people a feeling of mutual enjoyment, harmony and quiet (see "Fig. $1 ")$. 


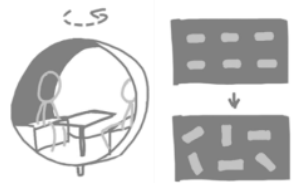

Fig. 1. Schematic program of Semi-open space.

4) Sinking space: The sinking space means that part of the room is sunk to create a clear, diverse and independent space in the overall interior space. Because the height of the sinking ground is lower than the surrounding height, it has a quiet and shaded character, making the sinking space a small world with a certain privacy. Under normal conditions, we should determine the drop height according to site conditions and usage requirements. The space is usually found in the reception area, the rest area of the coffee shop, and so on (see "Fig. 2").

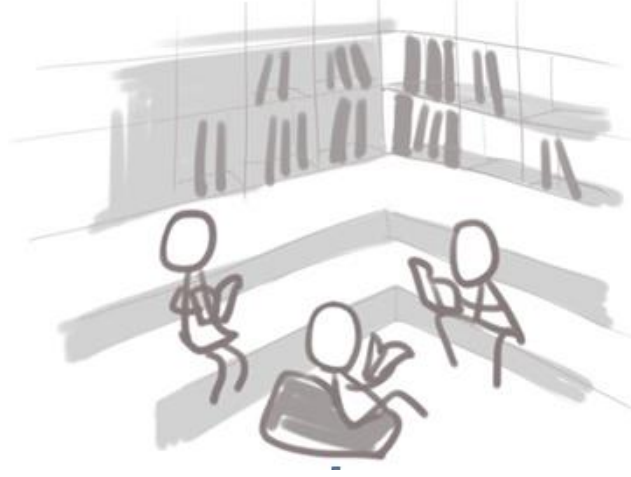

Fig. 2. Schematic diagram of sinking space.

5) Floor space: The platform refers to the ground that is partially raised in the space. It not only divides the indoor function, but also can be set as a storage space under the platform to effectively utilize the interior space. Compared with the surrounding space, the platform is prominent, and becomes the focus of the line of sight. At the same time, the platform space has the characteristics of storage, fun entertainment and display. The people on the platform are extremely wide and can give a very interesting taste. So the floor space is suitable for eye-catching furnishings or lookouts. The floor is set at an appropriate position in the interior space. The space below the platform has other functions such as storage and ventilation, which not only improves the indoor environment, but also makes the space rich and interesting (see "Fig. 3").
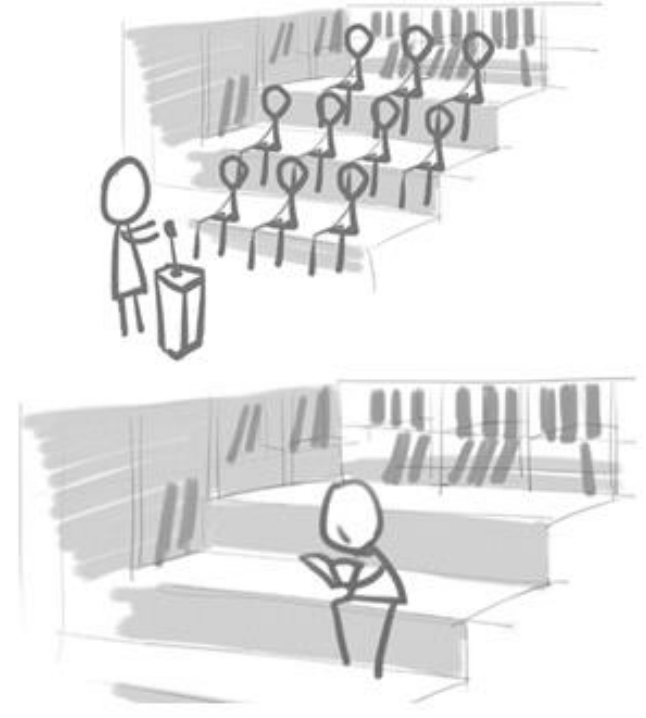

Fig. 3. Schematic program of Ground space.

6) Psychological space: Psychological space means that the space is not divided by the actual shape and material, and relies on the color or shape to associate or feel the space. Because of the lack of strong restrictions and the division of space according to "visual reality", it is also called "virtual space." For example, the floor and ceiling can be raised or lowered locally, or the space can be defined by variations in materials and colors. The space is used usually in the overall space, communicates with the whole space, but has a certain sense of scope and individuality at the same time. It is usually formed with the help of various spaces, displays, furniture, lighting, colors, materials and other factors.

\section{B. Design Principles for Interior Spaces}

1) Interior space design based on environment and use: The interior space design is based on the architectural design. The architectural design usually is a overall design process of of the entire building frame including the rough design of the interior space, the architectural design and so on. Design is the process of changing interior space and usually people spend most of their time in interior space. Therefore, the design of interior space brings more effects for people's daily life and work which means interior design is more important. Environmental factors have a certain impact on interior space design and architectural design. For example, the orientation and layout of the building, the terrain and traffic of the surrounding environment will have certain effects on the architectural design. In addition, the choice of decorative materials will have a certain effect on the interior space design. In the design process of interior space, after fully considering environmental factors and the use of interior space, the interior space can be modified to achieve the use and special needs of the space.

2) Adhering to the overall design thinking: Interior space design is the main component of architectural design. 


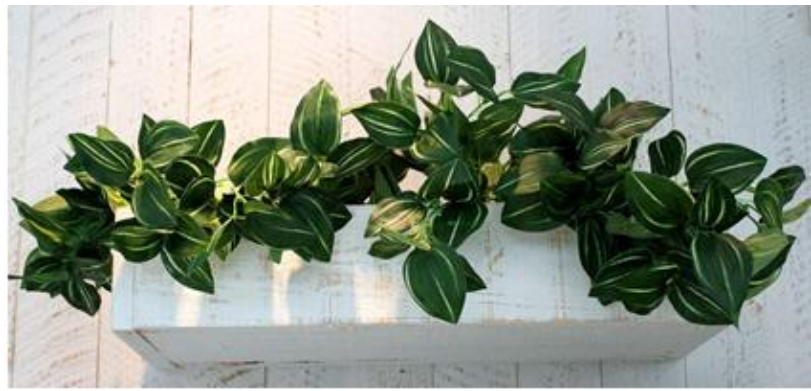

Fig. 4. Green plant.

2) Flexible design in interior space design: At present, more and more people like to choose small-sized living space, so the scalability of the room has become a factor that must be considered. For example, when families are starting renovations, most of the bay windows have been changed by placing the drawers below to save a large part of the space which is a more flexible decoration method. For example, in some single apartments, the bed is designed as a folding sofa bed, which can be folded and stored when not needed, and unfolded again when needed, so that the space can be fully utilized. In interior design, flexible design can start with four elements: point, line, face and body. These four basic elements can create different styles of space: point - the simplest form of visual art, giving the most flexible visual experience. Because the point has the characteristics of centripetal force and center of gravity, it can become the focus in space design. Lines - the lines in the art design are slender and sharp, so you can combine the lines with different features to create a visual impact. For example, the straight line is mainly used in the regular square space, which is simple and generous and is favored by young people. The curve gives a soft feeling. Face consisting of multiple points and multiple lines and with dynamic features. The face has a variety of emotional charm, so the face will give people a different visual impact. Body - being a more stereoscopic and intuitive art form, It is a collection of science, technology and art. A body is composed of various elements such as crafts and materials. In space design, we must combine body, faces, lines, points, and many other elements to create a personalized space.

\section{RE-RECOGNITION OF BUILDING COMPLEX} green plants are more lively and attractive than any other display, so it is appropriate to add some green plants to the interior space design. Each plant has a different color and shape. Therefore, the use of indoor greening can contribute to the formation or adjustment of the ecological space, and can implicitly dividing the space, making the whole indoor environment more harmonious and interesting. At the same time, you can also choose some green soft decorations which are made of green linen for the space, such as table, chairs, tablecloths, etc., to help to create a livelier environment (see "Fig. 4").

\section{A. Types of Building Complexes}

There are several types of building complexes: residential complexes, office conference complexes, transportation hub complexes, cultural and entertainment complexes, and commercial complexes.

The residential complex is mainly based on residential functions and is complemented by a retail complex of retail services, restaurants, sports venues, swimming pools, cinemas, parking lots and roof gardens; the office conference complex is a comprehensive centre providing office space, exhibition, conference and information exchange services; transportation hub complex has interchange hubs with 
domain and security, which can bring people together, all of which makes the city have a strong sense of life. with accommodation, catering and retail services while efficiently and conveniently evacuating people; cultural and entertainment complex is versatile complex integrating performance, entertainment, fitness, appreciation and dining together; the commercial complex brings together shopping malls or commercial streets of all types of large shopping malls, specialty stores and retail stores, usually with food, entertainment and leisure and other functional content.

\section{B. The Layout of the Building Complex}

The building complex has the following common layouts: channel, hall, axis, and cluster.

Channel type means that the pedestrian street is built on the direction of a straight road. Each space is listed on one side or both sides of the road. The passage is obvious and the guidance is stronger. The hall type means that there are other assisting functional facilities crosswise placed around the hall; the axis type is a spatial form that can give the building space a rhythm and sequence, and create a visual center, which can accommodate inconsistent forms and spaces with various forms together to create a complete and unified spatial rule; the cluster type means that the artistic charm of a single building in a city, especially for people, is much lower than that of a building group. Because the group effect has a certain effect, and the interaction between individuals has greatly increased the charm of this whole building groups, the image characteristics can be highlighted by improving the attraction and sensibility of the building image.

\section{Application Advantages of Building Complexes}

1) Efficient space-time utilization: In contrast to buildings that used to have only one function, building complexes have significant advantages in space applications. Its site utilization is repetitive and multi-layered. It utilizes space multi-functionality to achieve the goal of abundant use of urban space, which can save more urban space compared with the independent design of various activities above. The coexistence of multiple functions of the building group is conducive to improving urban efficiency. In a building complex, people can carry out multi-functional activities to meet basic living needs, which helps to alleviate traffic congestion and save people a lot of time.

2) Effective self-regulation mechanism: Due to the complexity of the functions of the building complex, it has many links with society and is able to better grasp the key points of changed social needs. When social needs change, the building complex can adjust itself to meet new demands. When better conditions are provided by the outside world, the building complex will operate in good condition, which will greatly enhance the development potential of each part of the building complex.

3) Good environmental value: Building complexes can organically organize diverse urban life to promote for each other. The multi-performances of the building complex make the urban residents' life rich and convenient. At the same time, the environment gives people a stronger sense of

\section{TAKING INTERIOR DESIGN RENOVATION OF NEW COMPOSITE ENTREPRENEURIAL SPACE AS EXAMPLE}

In the 21st century, mass entrepreneurship has become the background of this era. The main group of entrepreneurs has transitioned from the post-80s to the contemporary college students, and the number is huge. At the same time, the Beijing has become one of China's five major entrepreneurial centers. The entrepreneurial space has become an important part of a city, and will advance with the development of the city. Therefore, the design content proceeds from the aspect of entrepreneurship, and take the entrepreneurial group as the design object. Finally, the above specific theoretical research on the interior space of the building complex should be applied into the design.

\section{A. Satisfaction of Functional Requirements}

Design is an art serving people and should be used by people to meet people's spiritual and physiological needs. The emergence of building complexes gives the characteristics of spatial diversity, and people can expect to seek a sense of belonging within them. Its versatility requires that in the design of the space, in addition to meeting the material needs of people, it is also necessary to meet the spiritual needs of people. For example, the size of the negotiation room and office, the height and width of the desk in the office, the height of the seat, the brightness adjustment of the lighting, the choice of color, etc., should firstly meet the functional requirements. Then based on the most basic use functions, how to use human design to create a more comfortable space should be considered.

\section{B. Definition of Spatial Form}

The internal types of building complexes are various, which can satisfy the various use needs of the entrepreneurial group. The reasonable and effective grasp of the spatial scale and scale connection has a very important influence on shaping the interior space.

Due to the different functions of the building, the requirements for the scale in the internal space are different. The scales required for the atrium, the office area and the conference room are larger, and the open kitchen, fitness area, accommodation space and other types of space are smaller. The low and narrow space environment can create a sense of oppression in people's minds, and that a tall and spacious space can release people's mind and feel comfortable from the heart.

The interior of the building complex has a richly shaped space, some of which are regular and some are irregular. Regular shapes such as lines, squares, and circles can give a sense of quietness and stability. Irregular shapes can affect a person's visual perception, cause certain shocks and psychological fluctuations, resulting in emotional changes. In order to avoid the boring caused by a single spatial form, it should arrange the rule space and the irregular space in a crossed way to improve the interest of the space. 
The study choices building group of the Zhonghe Village in the courtyard to be transformed, sited at the Yuantong Bridge in the East Fifth Ring Road in Beijing as the object. Because when people mentioned Beijing architecture, in addition to the royal architecture of the Forbidden City, Yuanmingyuan, and the Summer Palace, which was first thought of, it was Beijing's local characteristic building, the bungalow Siheyuan. Siheyuan is a street space that is different from physical buildings and has an essential space combination function. Therefore, whether the physical function or cultural symbols, the Siheyuan has a great influence and significance for Beijing (see "Fig. 5"). Although the Siheyuan is different from the huge architectural complex mentioned in the past, its internal functions are completed and can know the actual design meaning of the building complex from the point to face.

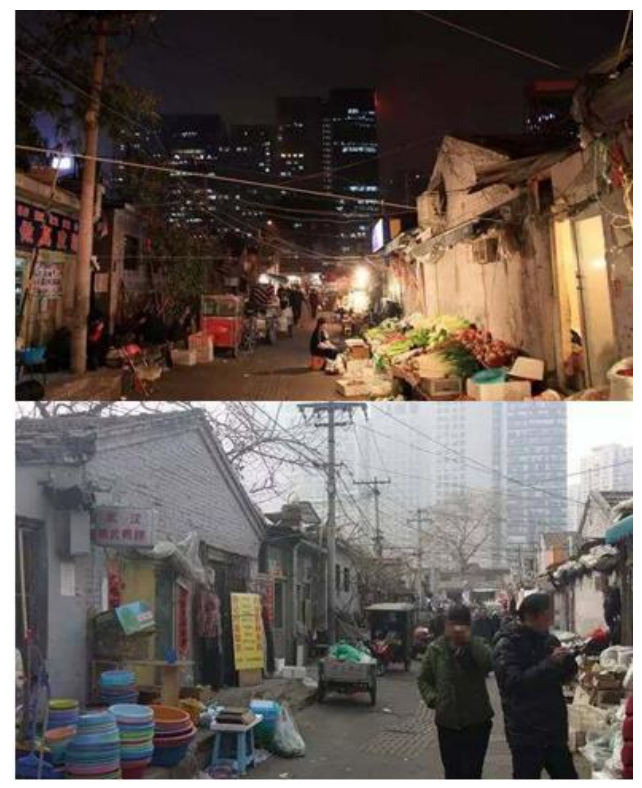

Fig. 5. The current situation of the village in the city.

In this study, the spatial processing methods of "surrounding" and "penetrating" were mainly adopted. "surrounding" and "penetrating" are an art form in the composition of Chinese painting. Appropriate use of the method can make the picture more vivid and create a freehand picture effect. In the design, the interior space can be processed by the division of space, such as closed, semiopen, partial divided, etc. The concepts and techniques of "surrounding" and "penetrating" are reflected in it. The characteristics of the "surrounding" and "penetrating" of the Siheyuan itself are conducive to the richness of the spatial form.

When people work together, talk or hold other activities in a relatively empty space, they often feel bothered and lack privacy, and the intimacy among people is not enough. Although the closed space can alleviate the above deficiencies, it is also prone to feelings of depression, clogging and crowding at work. Therefore, the space method of "surrounding" and "penetrating" is extremely important here. It is a secondary definition of a space. In the study, three main design methods of semi-open space, sinking space and lifting space are adopted which actually use physical or symbolic means to define a small space in the original space to create a new space atmosphere (see "Fig. 6").

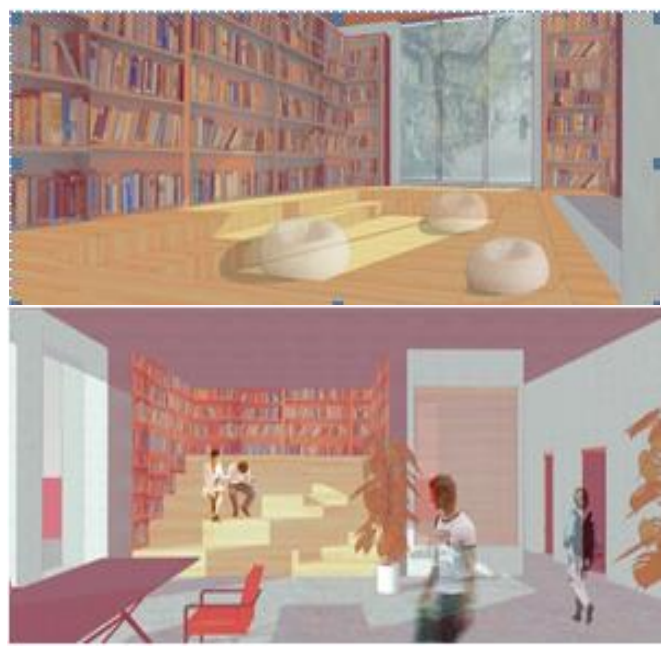

Fig. 6. Schematic diagram.

\section{Creation of Environmental Atmosphere}

In a certain environment, for the entrepreneurial groups, their basic work space requirements should be met. The basic design principle of the humanized design of the interior space of the building complex is to ensure the comfort of the indoor work space atmosphere to meet the basic physiology which make people do not feel depressed or tired. Perfect facilities and comfortable space environment can better attract entrepreneurs, enable entrepreneurs to stay and conduct activities in the space environment for a long time. The scale, functional layout, activity streamlines, daylighting and illumination, ventilation, and temperature regulation of the interior space all have an extremely important impact on human comfort. Designers can create a green and ecological indoor office space environment through the arrangement of green plants and landscape facilities. Introducing nature into the interior space can blur the boundaries of space and break the traditional working atmosphere.

\section{Reproduction of Cultural Connotations}

As a design method of interior space, humanized design not only reflects functional problems, but also represents social and cultural issues. As part of the organic whole of the building, the humanized design of the interior space environment of the complex should have its own personality, so it can also have cultural characteristics. For example, the folk color of the nation can directly reflect the national culture. In the long-term growth process, many countries in the world have produced a unique color culture, such as the five colors of yellow, cyan, red, black and white in contrast to the Chinese Five Elements Theory; Japan's opposite red, black, white, and cyan colors; ancient Irish symbolic color: black (north), white (south), purple (east), dark brown (west) and so on. Applying these colors to the design of the interior 
space environment inspires people's cultural identity and sense of belonging, reflecting the importance of using culture as a design guiding principle. The continuous development of social history and culture has led to the gradual formation of humanized design, and its cultural essence is obvious.

\section{CONCLUSION}

The building complex is a comprehensive and complex system involving all aspects of society, economy, culture, science and technology, life and so on. The diversified business layout and the satisfaction of multiple functional requirements make it present good competitiveness and unlimited vitality in the fast-growing economic trend. Based on the theory of interior space design, through the research on the status of building complex, the interior design of the ecological building complex is further controlled.

\section{A. Formal Beauty}

In the process of creating beauty, people form a summary and abstract summary of the style rules dividing into three categories: symmetric equilibrium, coordinated comparison and diverse unity. The presentation of the form is the carrier of design concept and the artistic conception of an interior space. The form coordination and aesthetics of the interior space of the building are the embodiment of the form beauty of the space design. Functionality is the first in interior design. While maximizing the use of design objects, it should meet the basic requirements of form beauty. Blindly focusing on practical functions can lead to the loss of original meaning of interior design and the inability to satisfy people's spiritual pursuits. On the contrary, if you only pay attention to the form beauty but do not consider the use of functional design, it is tantamount to putting the cart before the horse. Therefore, we must correctly handle the relationship between the form beauty and the functional design. Only by properly understanding and grasping the degree between the form beauty and the functional design can human emotions be given to substances that are not originally emotional.

Different from the traditional form beauty of interior space design, it is only a separate external form definition. Now people need to combine the use functions and the times characteristics with the aesthetic needs. A more meaningful interior space design based on the use of space functions can be achieved.

\section{B. Cultural Reconstruction}

In the 21 st century, with the development of the breadth and depth of human interaction, the speed of mutual integration and inter-cultural penetration has become faster and faster, and the scale has become larger and larger. Under the influence of Western culture invading China at an alarming rate, young people seem to be more and more strange to Chinese traditional culture. In the face of the "post-colonial" phenomenon that is now widespread, we have to do a good job. In the context of the current globalization of culture, how to design an ecological space that is combined with the times and has cultural characteristics is a challenge we need to accept.

The integration of man and nature is a clear and prima facie in the process of the growth of Chinese traditional culture. It is believed that the development of human beings and the development of nature are mutually influential. Therefore, in the design of architecture, people also pay attention to perfect integration of space and nature. In fact, the design development of different countries is a process of transitioning from national design style to modern design style, and then highlighting the cultural symbols of their national characteristics on the basis of modern style.

The modernist style is an architectural decoration that emphasizes breaking through tradition, promoting simplicity and opposing excess. The standard style has a great influence on traditional national and regional characteristics, resulting in the same style of interior space design. Although the modernist style satisfies the functional requirements to the greatest extent, it ignores the inner spiritual needs of people and discards the unique affinity of national culture. Therefore, we should adopt the attitude of "the essence to its dregs" in the treatment style of the design style in West, and strive to find the matching point of different styles of Chinese and Western cultures, instead of blindly copying the Western design works. The two processes of reference and innovation are indispensable. Only by mediating the relationship between the reference and innovation can we make excellent design works. If we want to make more interior space design works that match Chinese characteristics, we must grasp the relationship between modern style and traditional elements. The proper coexistence of the modern style and traditional elements is the basis of China's interior design.

\section{Being People-oriented}

In today's society, most people spend most of their time indoors, so the form beauty and cultural inner feelings and evaluations of interior space design come from people living in the space. People expect their designers and hope to improve their living and working environment with their designs. Therefore, the "people-oriented" design concept is particularly important.

Interior design is not only an artistic behavior, but also an expression of emotion among people. Designers and users can use their work to complete emotional communication. The designer uses his design to show his emotions while also satisfying the user's emotional needs. Although it is easy to ignore the feelings brought by space because of the habit of the space, the space does affect our emotional activities subtly.

Therefore, creating a living environment where person is willing to stay here and can obtain comfort and enjoyment is the ultimate goal of interior space design. While the world is constantly developing, people are constantly making progress. Although our needs for interior space design are constantly changing, the design concept based on people's activity needs is long-lasting and unchanged. 


\section{REFERENCES}

[1] Design combined with city — architectural complex design analysis. Kunming University of Science and Technology. 2002.

[2] "He" Contemporary Urban Building Complex Research. Tongji University. 2008.

[3] Urban architecture complex design research under symbiosis theory. Xiamen University. 2007.

[4] Time and Space Docking: Construction Strategy of Building Complex in Central District of Suzhou under TOD Mode. Planner. 2015.

[5] Preliminary study on the design of building complex based on urban space theory. Central Academy of Fine Arts.2016.

[6] Research on communication space design strategy of cultural architecture complex. South China University of Technology. 2012.

[7] Research on the integration design of building complex and urban public space. China University of Mining and Technology.2014.

[8] Urban residential complex and its adjustment to urban spatial structure. Fujian Architecture. 2002.

[9] Research on the leading function positioning of urban complexes in China. Zhengzhou University. 2014.

[10] Tokyo Daikanyama Residential Complex, Japan. World Architecture.1996. 